\title{
Oscillating Sign of Drag in High Landau Levels
}

\author{
Felix von Oppen ${ }^{1}$, Steven H. Simon ${ }^{2}$, and Ady Stern ${ }^{3}$ \\ 1 Institut für Theoretische Physik, Freie Universität Berlin, Arnimallee 14, 14195 Berlin, Germany \\ ${ }^{2}$ Lucent Technologies, Bell Labs, Murray Hill, NJ, 07974 \\ 3 Department of Condensed Matter Physics, The Weizmann Institute of Science, 76100 Rehovot, Israel
}

(April 30th, 2001)

\begin{abstract}
Motivated by experiments, we study the sign of the Coulomb drag voltage in a double layer system in a strong magnetic field. We show that the commonly used Fermi Golden Rule approach implicitly assumes a linear dependence of intra-layer conductivity on density, and is thus inadequate in strong magnetic fields. Going beyond this approach, we show that the drag voltage commonly changes sign with density difference between the layers. We predict that in the Quantum Hall regime the Hall and longitudinal drag resistivities are comparable. Our results are also relevant for pumping and acoustoelectric experiments.
\end{abstract}

Drag experiments in coupled two-dimensional electron systems provide information on the response of a system at finite frequency and wavevector and are thus complementary to standard DC transport measurements [1,2]. In a typical drag experiment, a current is applied to the active layer of a double-layer system and the voltage $V_{D}$ induced in the other passive layer is measured, with no current allowed to flow in that layer. In a simple picture of drag, the current in the active layer leads - via interlayer Coulomb or phonon interaction - to a net transfer of momentum to the carriers in the passive layer. At conditions of zero current in the passive layer, this momentum transfer is counteracted by the build-up of the drag voltage $V_{D}$. In the cases of two electron layers or two hole layers, the drag voltage points opposite to the voltage drop in the active layer. This is defined as positive drag. Negative drag occurs in systems with one electron layer and one hole layer [2, 3].

Prior theoretical work on drag [2, [4, 5] was often based on or reduced to a Fermi Golden Rule analysis (see, e.g., Zheng and MacDonald [1]). In this analysis, the sign of drag does not vary with magnetic field $B$, temperature $T$, or difference in Landau level (LL) filling factor $\nu$ between the two layers. By contrast, recent experiments at large perpendicular $B$ [6] observe that the sign of drag changes with all of these parameters in systems of two coupled electron layers - specifically in the Shubnikovde-Haas ( $\mathrm{SdH}$ ) and integer quantum Hall (IQH) regimes. Feng et al. [6] find positive drag whenever the topmost partially filled LLs in both layers are either less than half filled or more than half filled. Negative drag is observed when the topmost LL is less than half-filled in one layer and more than half-filled in the other. Even more surprisingly, Lok et al. [7] maintained that the sign of drag was sensitive to the relative orientation of the majority spins of the two layers at the Fermi energy.

In this paper, we show that the use of the Fermi Golden Rule approach is inappropriate in the $\mathrm{SdH}$ and $\mathrm{IQH}$ regimes and that a more careful analysis opens different routes to drag with changing sign. Remarkably, we find that Hall drag can be of the same magnitude as longitudinal drag in these regimes. We emphasize that a naive rationale for the experimental results of Feng et al. which points to the similarity between a less (more) than half-filled Landau level and an electron (hole) like band - leaves out essential physics of the problem. Finally, we compare our results to the existing measurements [6,7] and propose some interesting experiments.

We start from a general linear-response expression [2] which relates the drag conductivity $\hat{\sigma}^{D}$ to the rectification coefficients $\boldsymbol{\Gamma}(\mathbf{q}, \omega)$ of the active and passive layers,

$$
\begin{aligned}
\sigma_{i j}^{D}=\int \frac{d \omega}{2 \pi} \sum_{\mathbf{q}} \frac{|U(\mathbf{q}, \omega)|^{2}}{8 T} & \sinh ^{2}(\omega / 2 T) \\
& \times \Gamma_{i}^{p}(\mathbf{q}, \omega ; B) \Gamma_{j}^{a}(\mathbf{q}, \omega ;-B) .
\end{aligned}
$$

The drag conductivity gives the current response $\mathbf{J}^{p}$ in the passive (dragged) layer via $J_{i}^{p}=\sigma_{i j}^{D} E_{j}^{a}$ to an applied electric field $\mathbf{E}^{a}$ in the active layer. Its relation to the commonly measured drag resistivity is clarified below. In Eq. (11), $U(\mathbf{q}, \omega)$ is the screened interlayer (Coulomb or phonon-mediated) interaction and the rectification coefficient $\boldsymbol{\Gamma}(\mathbf{q}, \omega)$ is defined by

$$
\mathbf{J}^{d c}=\frac{1}{2} \sum_{\mathbf{q}, \omega} \boldsymbol{\Gamma}(\mathbf{q}, \omega)|e \phi(\mathbf{q}, \omega)|^{2} .
$$

In Eq. (22), $\mathbf{J}^{d c}$ is the DC current induced in quadratic response to the driving force exerted by a screened potential $\phi(\mathbf{q}, \omega)$ of wave-vector $\mathbf{q}$ and frequency $\omega$.

The physical interpretation of the drag expression Eq. (11) is that the voltage in the active layer creates an asymmetry (between $\mathbf{q}$ and $-\mathbf{q}$ ) in the thermal density fluctuations in that layer. These fluctuations are transferred to the passive layer - via Coulomb or phonon interaction 
- where they are rectified to create a current. In experiment, usually the drag resisitivity $\hat{\rho}^{D}$ is measured, which gives the electric field response $\mathbf{E}^{p}$ in the passive layer to a current $\mathbf{J}^{a}$ driven in the active layer via $E_{i}^{p}=\rho_{i j}^{D} J_{j}^{a}$. For weak drag, it is related to the drag conductivity by

$$
\hat{\rho}^{D}=\hat{\rho}^{p} \hat{\sigma}^{D} \hat{\rho}^{a}
$$

where $\hat{\rho}^{p(a)}$ is the resistivity tensor of the passive (active) layer.

A simple but instructive approach to rectification considers situations in which current and field are locally related, $\mathbf{J}(\mathbf{r}, t)=-\hat{\sigma}(n(\mathbf{r}, t)) \nabla \phi(\mathbf{r}, t)$, and the conductivity depends on position and time only through the local density $n(\mathbf{r}, t)$ [9]. In addition to the current, the perturbation $\phi(\mathbf{q}, \omega)$ also induces a density perturbation $\delta n(\mathbf{q}, \omega)=\Pi(\mathbf{q}, \omega) e \phi(\mathbf{q}, \omega)$ due to the polarizability $\Pi(\mathbf{q}, \omega)$. Up to quadratic order in the applied potential,

$$
\mathbf{J}(\mathbf{r}, t)=-\left[\hat{\sigma}\left(n_{0}\right)+\frac{d \hat{\sigma}}{d n} \delta n(\mathbf{r}, t)\right] \nabla \phi(\mathbf{r}, t) .
$$

Taking the time and space average of the second term then yields a DC contribution to the current given by

$$
\mathbf{J}_{d c}=\sum_{\mathbf{q}, \omega}\left(\frac{d \hat{\sigma}}{d n}\right)[\Pi(\mathbf{q}, \omega) e \phi(\mathbf{q}, \omega) i \mathbf{q} \phi(-\mathbf{q},-\omega)]
$$

or equivalently the rectification coefficient is given by

$$
\boldsymbol{\Gamma}(\mathbf{q}, \omega)=-2 \frac{d \hat{\sigma}}{d(e n)} \cdot \mathbf{q} \operatorname{Im} \Pi(\mathbf{q}, \omega) .
$$

At zero magnetic field, the conductivity is local when considering scales large compared to the mean free path $\ell_{\mathrm{el}}$, implying that Eq. (6) holds in the diffusive regime defined by $q \ell_{\mathrm{el}} \ll 1[10$. At finite $B$, and for short range disorder, Eq. (6) is still valid in the diffusive regime $\omega \tau, D q^{2} \tau \ll 1$, where $D$ and $\tau$ denote the appropriate diffusion constant and scattering time. Indeed, we have checked this [11] by an explicit diagrammatic calculation in the self-consistent Born approximation (SCBA) [12]. Specifically, once the magnetic field is strong enough such that the cyclotron radius $R_{c}=\hbar k_{\mathrm{F}} /(e B)$ is small compared to $\ell_{\mathrm{el}}$ and for short-range correlated disorder, the conductivity is local on scales larger than $R_{c}$, and Eq. (6) holds for $q R_{c} \ll 1$ [10]. Since $R_{c} \ll \ell_{\mathrm{el}}$ even for very small magnetic fields, and since the diffusion constant decreases rapidly with the application of a magnetic field, Eq. (6) is expected to be applicable for a significant part of the wave-vector range of the integration in (11) for typical experiments.

Using Eq. (6) in the general drag expressions, Eqs. (11) and (3), one readily finds that up to an overall positive prefactor, the drag resistivity tensor becomes

$$
\hat{\rho}^{D} \sim \hat{\rho}^{p} \frac{d \hat{\sigma}^{p}}{d(e n)} \frac{d \hat{\sigma}^{a}}{d(e n)} \hat{\rho}^{a} .
$$

This expression easily reproduces some standard results. In the absence of a magnetic field, the tensor structure is trivial. Observing that the conductivity increases (decreases) with increasing electron density for electron (hole) layers, we recover that coupled layers with the same type of charge carriers exhibit positive drag, while coupled electron-hole layers have a negative drag resistivity. If the system is strictly electron-hole symmetric, the derivative of the conductivity with respect to density vanishes and consequently, there is no drag. Treating the conductivity in the presence of a magnetic field in a simple Drude-type picture, $\hat{\sigma}$ depends linearly on the density en (so long as the scattering time is taken to be density independent). Eq. (6) then reproduces results of previous works [1,2,5] that calculate drag in a Fermi Golden Rule or scattering time approximation. In particular, in this approximation it is immediately found from Eq. (7) that the drag resistivity is diagonal and Hall drag vanishes identically. Finite Hall drag can appear even in a Drudetype approximation once the scattering time is taken to be energy and thus density dependent [13.

Interesting new effects appear in the presence of a strong magnetic field, in the $\mathrm{SdH}$ and $\mathrm{IQH}$ regimes, where the derivative of $\sigma_{x x}$ changes sign as the magnetic field or Fermi energy is varied. While this is superficially quite reminiscent of the experimental results of Refs. [6, 7], the details can be involved due to the many terms that contribute, once the tensor products in Eq. (7) are multiplied out. In the experimental samples, typically $\rho_{x y} \gg \rho_{x x}$ already at very small magnetic fields. Thus, up to an overall positive prefactor

$$
\rho_{x x}^{D} \sim \rho_{x y}^{p}\left\{\frac{d \sigma_{y y}^{p}}{d(e n)} \frac{d \sigma_{y y}^{a}}{d(e n)}+\frac{d \sigma_{y x}^{p}}{d(e n)} \frac{d \sigma_{x y}^{a}}{d(e n)}\right\} \rho_{y x}^{a} .
$$

Generally the derivative of the longitudinal conductivity $\sigma_{x x}$ changes sign in the $\mathrm{SdH}$ and IQH regimes, being positive for less than half filling of the topmost LL and negative for more than half filling. By contrast, the Hall conductivity generally increases monotonically with $n$, and its derivative is therefore positive.

It is not clear, apriori, which of the two terms in the curly brackets of Eq. (8) dominates. We obtain an oscillatory sign of drag, similar to the experimental results in Ref. [6] when $d \sigma_{x x} / d(e n)$ dominates over $d \sigma_{x y} / d(e n)$. However, if that were the case, drag would be negative for equal filling of the two layers (because $\left.\rho_{x y}=-\rho_{y x}\right)$, in contrast to the experimental observations. Positive drag for equal filling is only obtained when $d \sigma_{x y} / d(e n) \gg d \sigma_{x x} / d(e n)$. Were that the case, however, there would presumably be no sign changes of the drag resistivity. In fact, in the IQH regime the derivatives of both components of the conductivity tensor are experimentally of the same order (both change by approximately $e^{2} / h$ in the region of the plateau transition). While neither of the two limits is therefore realized in 
experiment, it is clear that Eq. (8) makes it difficult to obtain both positive drag for equal densities and a drag sign that oscillates with the difference in densities.

A striking consequence of Eq. (7) is that if we assume that $d \sigma_{x x} / d(e n)$ and $d \sigma_{x y} / d(e n)$ are of comparable magnitude in the integer-quantum-Hall regime then the Hall drag is of the same order as longitudinal drag. In fact, we have

$$
\rho_{x y}^{D} \sim \rho_{x y}^{p}\left\{\frac{d \sigma_{y y}^{p}}{d(e n)} \frac{d \sigma_{y x}^{a}}{d(e n)}+\frac{d \sigma_{y x}^{p}}{d(e n)} \frac{d \sigma_{x x}^{a}}{d(e n)}\right\} \rho_{x y}^{a} .
$$

Moreover, Hall drag generally changes sign with filling factor difference between the two layers.

Although the experimental disorder potential is long ranged, it is instructive to consider also the case of short-range disorder, for which the SCBA 12] becomes exact in the limit of high LLs [14]. Assuming that the disorder broadening is small compared to the LL spacing, the conductivity tensor $\hat{\sigma}$ becomes [12] $\sigma_{x x}=$ $\left(e^{2} / \pi^{2} \hbar\right) N\left\{1-\left[\left(E_{F}-E_{N}\right) / 2 \gamma\right]^{2}\right\}$ and $\sigma_{x y}=-(e n / B)+$ $\left(e^{2} / \pi^{2} \hbar\right)\left(2 \gamma / \hbar \omega_{c}\right) N\left\{1-\left[\left(E_{F}-E_{N}\right) / 2 \gamma\right]^{2}\right\}^{3 / 2}$ where $\gamma$ denotes the LL broadening, and the Fermi energy $E_{F}$ is assumed to lie within the $N$-th LL (of energy $E_{N}=$ $(N+1 / 2) \hbar \omega_{c}$ in the clean case). Thus $\left|d \sigma_{x x} / d(e n)\right| \gg$ $\left|d \sigma_{x y} / d(e n)\right|$ and $d \sigma_{x x} / d(e n)$ changes sign as a function of filling - being positive for less than half filled LLs and negative for more than half-filled LLs. Neglecting the derivatives of the Hall conductivity, one obtains for the diagonal drag resistivity of an isotropic system

$$
\rho_{x x}^{D}=C_{x x}(B, T) \frac{d \sigma_{x x}^{p}}{d(e n)} \frac{d \sigma_{x x}^{a}}{d(e n)}\left[\rho_{x x}^{p} \rho_{x x}^{a}-\rho_{x y}^{p} \rho_{x y}^{a}\right]
$$

with $C_{x x}$ a positive function. Thus, we again find the surprising result that drag is negative for equal densities in the two layers (since $\left|\rho_{x y} / \rho_{x x}\right| \geq \pi$ ). More generally, drag oscillates with difference in density between the layers. It is negative whenever the topmost occupied LLs in the two layers are both less than half filled or both more than half-filled. It is positive if the topmost LL is less than half-filled in one layer and more than half-filled in the other. Moreover, the Hall drag resistivity is comparable to the diagonal drag resistivity also in this model situation. In fact, one has

$$
\rho_{x y}^{D}=C_{x y}(B, T) \frac{d \sigma_{x x}^{p}}{d(e n)} \frac{d \sigma_{x x}^{a}}{d(e n)}\left[\rho_{x y}^{p} \rho_{x x}^{a}+\rho_{x x}^{p} \rho_{x y}^{a}\right],
$$

with $C_{x y}=C_{x x}$. Unlike before, this result is now a consequence of the fact that $\rho_{x x}$ and $\rho_{x y}$ are of similar magnitudes.

When the LL broadening is comparable to but exceeds the LL spacing $\left(\omega_{c} \tau<1\right)$, we find [1] from a numerical evaluation of the SCBA equations that by contrast, the derivative of $\sigma_{x y}$ dominates over that of $\sigma_{x x}$ while still changing sign as function of filling (for $N \gamma / \hbar \omega_{c} \gg 1$ ). However, in this limit, $\sigma_{x x}$ dominates over $\sigma_{x y}$ (and hence $\rho_{x x}$ over $\left.\rho_{x y}\right)$ so that again, we find negative drag for equal filling of both layers.

Experiments do not necessarily satisfy the condition $D q^{2} \tau \ll 1$ for the diffusive regime. Thus, we now turn to a discussion of drag in the ballistic regime where $D q^{2} \tau \gg 1$. At zero $B$, Eq. (6) holds also in the ballistic regime [2]. Indeed, Eq. (6) with $\hat{\sigma}$ given by the Drude expression is also derivable within a Boltzmann approach which should be valid for $q / k_{\mathrm{F}}, \omega / E_{\mathrm{F}} \ll 1$ and at fields low enough that $\mathrm{SdH}$ oscillations are absent [1]. Surprisingly, we find that this is not generally true for higher magnetic fields. While we have not succeeded in deriving a general expression, analogous to Eq. (6), in this regime, we computed the rectification explicitly in the high-magnetic field limit introduced above.

Our calculation starts from the diagrammatic expression for the rectification coefficient [2]

$$
\boldsymbol{\Gamma}(\mathbf{q}, \omega)=\frac{\omega}{\pi i} \operatorname{Tr}\left\{\mathbf{I} G^{-} e^{i \mathbf{q r}}\left[G^{-}-G^{+}\right] e^{-i \mathbf{q r}} G^{+}\right\}
$$

Here, $G^{ \pm}$denotes the impurity-averaged Green function in the SCBA, I is the current operator, and $\mathrm{Tr}$ the trace over the single-particle states. The calculation is simplified by the fact that vertex corrections can be neglected in the ballistic limit.

Using standard results for the matrix elements of the current and density operators between LL wavefunctions and exploiting the small parameter $\gamma / \hbar \omega_{c} \ll 1$, we obtain for the longitudinal rectification

$$
\Gamma_{\|}=\left(\frac{-4 \omega R_{c}}{N}\right) J_{0}\left(q R_{c}\right) J_{1}\left(q R_{c}\right)\left(\frac{d n}{d \mu}\right)^{2} \frac{d \sigma_{x x}}{d(e n)} .
$$

with $J_{i}$ the $i^{t h}$ Bessel function and $N$ the LL number. A similar calculation for the transverse rectification gives $\Gamma_{\perp}=\left(1 / \omega_{c} \tau\right) \Gamma_{\|}$or

$$
\Gamma_{\perp}=\frac{2}{3}\left(\frac{-4 \omega R_{c}}{N}\right) J_{0}\left(q R_{c}\right) J_{1}\left(q R_{c}\right)\left(\frac{d n}{d \mu}\right)^{2} \frac{d \sigma_{x y}}{d(e n)} .
$$

While both $\Gamma_{\|}$and $\Gamma_{\perp}$ still include factors that can be written as derivatives of the corresponding conductivity, similar to the diffusive regime, the prefactors are no longer equal to one another and cannot be expressed in terms of the polarization operator $\Pi(q, \omega)$. Remarkably, the rectification in the ballistic limit also changes sign with $q R_{c}$ due to the matrix elements of the density, in addition to the sign changes of $d \hat{\sigma} / d(e n)$ discussed above. Note that such behavior would be impossible in Eq. (6) since the sign of $\operatorname{Im} \Pi$, being fixed by the sign of $\omega$, does not change with $q$.

It is an interesting question whether these additional sign changes are reflected in the drag. This is not obvious since the oscillating Bessel functions depend on $q$ which needs to be integrated over to obtain the drag conductivity (cf. Eq. (1)). For Coulomb drag, the screened interlayer interaction can be approximated by the ThomasFermi result $U(\mathbf{q}, \omega)=\pi e^{2} q /\left(\kappa_{a} \kappa_{p} \sinh q d\right)$ with $\kappa_{a}\left(\kappa_{p}\right)$ 
denoting the Thomas-Fermi momenta of the active (passive) layer and $d$ the interlayer distance. Thus, the $\omega$ and $q$ integrations in Eq. (11) factorize. One readily observes that the $q$ integration is not dominated by the upper limit in the relevant region $1 / R_{c} \ll q \ll 1 / d$, and thus there are no additional oscillations from the matrix elements in Coulomb drag. This result is also confirmed by straightforward numerical evaluation.

For phonon drag, the situation is slightly different. Using a qualitative approximation [5], $|U(q, \omega)|^{2} \approx$ $K q^{3} \delta(\omega-q v)$ with $v$ the sound velocity, the integrals in Eq. (11) yield an additional factor $T^{4}\left[g\left(q_{T}\left|R_{c}^{a}-R_{c}^{p}\right|\right)+\right.$ $\left.g\left(q_{T}\left|R_{c}^{a}+R_{c}^{p}\right|\right)\right]$ where $q_{T}=\left(T / T_{B}\right) k_{\mathrm{F}}$ with $T_{B}=2 \hbar v k_{\mathrm{F}}$ the Bloch-Grüneisen temperature (for typical samples $T_{B} \approx 10 \mathrm{~K}$ ). Here, $g(x)$ is a smooth function (which can be calculated analytically) that starts with $g(0)=1$ and decreases for small $x$ crossing zero at $x \approx .04$ then reaching a minimum of $g(.075) \approx-.37$ then increasing monotonically and exponentially towards zero. For all but the lowest temperatures, the term involoving $\left|R_{c}^{a}-R_{c}^{p}\right|$ dominates, and thus the overall sign of the drag changes when $q_{T}\left|R_{c}^{a}-R_{c}^{p}\right|=\left|\nu_{a}-\nu_{p}\right| T / T_{B} \approx .04$.

In passing, we note that while the sign changes of $\boldsymbol{\Gamma}(\mathbf{q}, \omega)$ with $q R_{c}$ in the ballistic limit seem to have only minor consequences for drag, they should be observable in other experiments of recent interest, namely in pumping [15] and in acoustoelectric experiments [9], which also measure the rectification coefficient $\boldsymbol{\Gamma}(\mathbf{q}, \omega)$ [16]. In pumping experiments, a potential $\phi(\mathbf{r}, t)$ is applied to the system which is typically made up of the sum of two potentials oscillating out of phase $\phi(\mathbf{r}, t)=$ $\phi_{1}(\mathbf{r}) \cos (\omega t)+\phi_{2}(\mathbf{r}) \cos (\omega t+\delta)$. Due to the symmetries of $\boldsymbol{\Gamma}(\mathbf{q}, \omega)$, the pumping current density is given by $\mathbf{J}^{d c}=(1 / 2) \sin (\delta) \sum_{\mathbf{q}} \boldsymbol{\Gamma}(\mathbf{q}, \omega) \operatorname{Im}\left[\phi_{1}(\mathbf{q}) \phi_{2}(-\mathbf{q})\right]$. In acoustoelectric experiments, acoustic waves sent through a (piezoelectric) crystal apply an "external" electric potential $\phi^{e x t}(\mathbf{q}, \omega)$ to the electrons in the system with $\omega=c q$ and $c$ the wave velocity. The driven electric current is given precisely by Eq. (22) where $\phi(\mathbf{q}, \omega)=$ $\phi^{e x t}(q, \omega) /[1-v(q) \Pi(q, \omega)]$ is the screened potential associated with $\phi^{e x t}(v(q)$ denotes the bare Coulomb interaction). We note that the acoustoelectric experiments of Ref. [9], which were carried out in the diffusive regime, have been successfully analysed using Eq. (6).

For the cases considered here, an oscillating sign of drag is always accompanied by negative drag for equal fillings. This apparent conflict with the experimental results of Feng et al. might be resolved in one of the following ways: (a) For long-range disorder and $\hbar \omega_{c}$ comparable to the LL broadening, the Hall conductivity might become non-monotonous, cf. Eq. (7) and the SCBA results. (b) In measurements, the large Hall drag resistivity might mix into the measured drag response. On the other hand, a simple-minded extension of our results to include the two spin directions definitely fails to account for the apparent spin dependence observed in Ref. [7]. We take this failure as an indication that the observations of Ref. [7] cannot be explained by theories where correlations induced by Coulomb interactions are neglected.

In conclusion, we have considered drag in high LLs with an emphasis on the sign of the effect. Remarkably, we find that both in the ballistic and the diffusive regimes, drag in high LLs cannot be described by a widely-used Fermi Golden Rule expression [1]. Our analysis naturally opens the possibility of sign changes of the drag resistivity as function of filling factor difference between the two layers. Moreover, it implies that Hall drag can be of the same order as longitudinal drag in high LLs. Surprisingly, we find that the sign of drag can be quite different from naive expectations. In particular, in several regimes drag is negative for two identical electron layers. We believe that it would be particularly interesting to check experimentally our predictions that Hall drag can be of the same order as longitudinal drag and that the acoustoelectric current in the ballistic regime changes sign as function of the wavevector.

We thank B.I. Halperin, K. von Klitzing, S. Lok, M. Raikh, U. Sivan, and Y. Yaish for instructive discussions. We acknowledge financial support from the USIsrael BSF, the Israeli science foundation, DIP-BMBF (AS), and through the DFG-Schwerpunkt "QuantenHall-Systeme" (FvO). FvO thanks the Einstein Center and the LSF at the Weizmann Institute for hospitality.

[1] L. Zheng, A. MacDonald, Phys. Rev. B, 48, 8203 (1993).

[2] A.Kamenev and Y. Oreg, Phys. Rev. B. 52, 7516 (1995); K. Flensberg et al., Phys. Rev. B 52, 14761 (1995).

[3] U. Sivan et al., Phys. Rev. Lett. 681196 (1992).

[4] K. Flensberg et al., Phys. Rev. B 56, 10314 (1997).

[5] M. C. Bonsager et al., Phys. Rev. B 57, 7085 (1998).

[6] X. G. Feng et al., Phys. Rev. Lett. 813219 (1998).

[7] J. G. S. Lok et al., cond-mat/0011017.

[8] U. Sivan, Y. Yaish, private communication.

[9] A. Esslinger et al., Surf. Sci 305, 83 (1994).

[10] Here we neglect phase coherence which leads to small nonlocal contributions on scales of the coherence length.

[11] F. von Oppen, S.H. Simon, and A. Stern, to be published.

[12] T. Ando, A. B. Fowler, and F. Stern, Rev. Mod. Phys. 54, 437 (1982) and references therein; we note that the SCBA is not applicable for long range disorder, as discussed in M. E. Raikh and T. V. Shahbazyan, Phys. Rev. B 47, 1522 (1993).

[13] B.Y.K. Hu, Phys. Scripta T69, 170 (1997).

[14] K.A. Benedict, J.T. Chalker, J. Phys. C 19, 3587 (1986).

[15] M. Switkes, C. M. Marcus, K. Campman, and A. C. Gossard, Science 283, 1905 (1999).

[16] M. G. Vavilov, V. Ambegaokar, and I. L. Aleiner, condmat/0008469. 